\title{
Modelling driver behaviour in the case of failures in a steer-by-wire system
}

\author{
TNO Human Factors \\ Traffic Behaviour \\ PO Box 23 \\ 3769 ZG Soesterberg \\ The Netherlands \\ jeroen.hogema@tno.nl
}

Jeroen Hogema \& Paul Wewerinke

\begin{abstract}
In the design and development of advanced vehicle control systems such as X-by-Wire (XBW), system safety is a crucial aspect. Failures in XBW can easily result in accidents. Therefore, methods and tools are needed to ensure fault-tolerant systems. Quantifying the consequence of an error is far from trivial, since the consequence is determined not only by the vehicle and the XBW system, but also by the driver's response. This chapter describes a driver model for the case of a Steer-by-Wire system. A rather good match between the model and the experimental results from a driving simulator study could be obtained for all configurations considered. The resulting model can be used to predict driver's response to tasks, similar to the type of failure tasks considered here, providing a useful method to answer a variety of design questions related to fault-tolerant system design.
\end{abstract}

\section{Introduction}

The 'by-Wire' technology - as in drive, brake and steer - introduces new possibilities optimising for handling and comfort. Selecting driver-specific settings becomes possible, and furthermore, 'by-Wire' systems can lead to reduced production costs and packaging advantages. At the same time, 'by-Wire' introduces new challenges in terms of system safety. A technical failure in a 'by-Wire' system can have severe safety consequences. Fault-tolerant design methods are needed to ensure that a single failure will not lead to a catastrophic event. This necessity is reflected in the development of FlexRay, a communication protocol for 
automotive applications, which has not only high performance, but also faulttolerance and redundancy as main features [1].

In general, both the probability and the consequences of system errors have to be considered. Obviously, errors with 'serious' consequences need to have a 'low' probability, whereas errors with 'innocent' consequences can occur with a 'somewhat higher' probability without endangering safety (although trust in the system may still suffer). However, quantifying the consequence of an error is far from trivial, since the consequence is determined not only by the vehicle and the bywire system, but also by the driver's response. Embedding the driver's response to such errors in a driver model will facilitate the design process.

This chapter describes driver model for the case of a system failure in steer-bywire systems. An existing validated driver model for the lane keeping task was used as the starting point. For a detailed description, the reader is referred to [2]. In parallel to the driver modelling work, a driving simulator study was to provide empirical data for calibration and validation of the driver model. The responses to the system errors were analysed in terms of the extremes of the path deviation, the yaw rate, the heading angle and the steering wheel angle.

\section{Method}

\subsection{Steer-by-wire and errors}

The Steer-by-Wire (SBW) system consisted of a conventional steering wheel as the input device and two main control systems: a rack actuator that controlled the wheel angle and a torque actuator to provide the driver with a steering wheel feedback torque. The steering wheel torque setpoint of the SBW system was based on a simple spring-damper system, i.e. with the torque setpoint proportional to the wheel angle and the wheel angle angular velocity. The wheel angle and the steering wheel angle were related via a simple gear ratio. Two error types are described in this chapter, both consisting of a pulse on the wheel angle, defined by their amplitude and duration. For the first error type ('Torque'), the error manifested itself in the vehicle motion and in the steering wheel torque. For the second error type ('No torque'), the error on the wheel angle manifested itself only in the vehicle motion, not in the steering wheel torque. Four conditions are included: the two error types, and two pulse durations (50 and $200 \mathrm{~ms}$ ), all with a fixed error amplitude of $2.3 \mathrm{deg}$. 


\subsection{Driving simulator study}

A driving simulator study was conducted in the high-fidelity driving simulator of TNO (see [4] for more details). In this study the participant was seated in a BMW 318I mock-up, which was placed on a motion base with six degrees of freedom. A high-quality control loader provided the steering wheel torque. The participant watched a large radial screen on which the environment was projected. The road environment in the experiment consisted of one straight lane of $3.40 \mathrm{~m}$ wide with solid markings on both sides and no obstacles along the road, without any other traffic.

Driving speed is a factor that affects the impact of a SBW error: the effect of a given error on the vehicle path is more severe as driving speeds increase [3]. Thus, the most critical situation is at high speeds. In the current experiment the driving speed was kept constant at $120 \mathrm{~km} / \mathrm{h}$.

To incorporate the driver's expectation of errors, we distinguished two separate groups. The first group ('expecting') consisted of 16 subjects who received multiple errors and who were informed that errors would occur. In total, there were 88 errors for each participant. The second group ('surprised') consisted of 36 subjects who each only received one error, without knowing beforehand that this would occur.

\subsection{Driver model}

The modelling approach was based on the Optimal Control Model structure, in line with the work of e.g. [5], based on a linear system theoretic approach. The fundamental hypothesis is that the human operator behaves optimally, according to a certain criterion, given his inherent limitations and constraints. A global diagram of the model components is shown in Fig. 1.

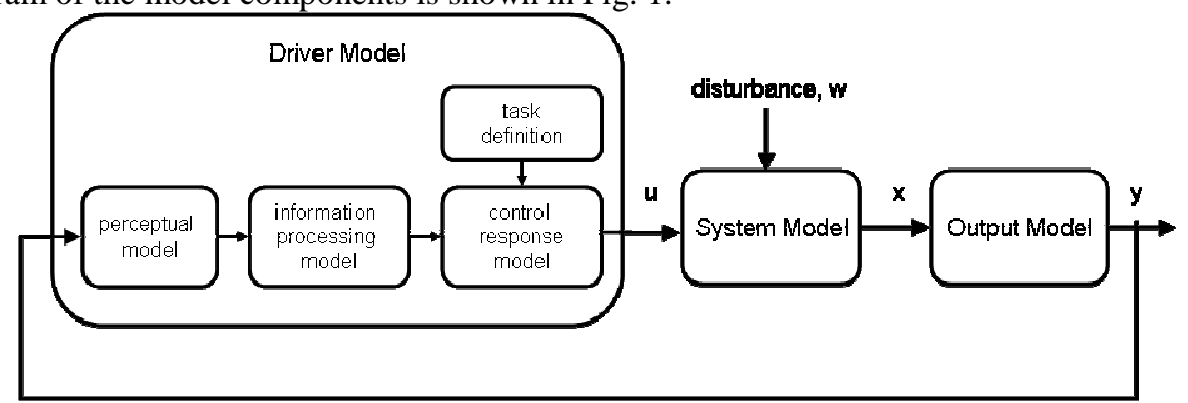

Fig. 1 Overview of the model.

The System model describes the dynamics of the system controlled by the driver. A linear model formulation is used. The system state $x$ is related to the 
driver control input $u$ (in this case the steering wheel torque). The system state is also influenced by the system disturbance $w$, which includes the deterministic system errors as well as random inputs to account for wind, road surface effects, etc.

The output model determines the variables that are perceived by the driver $(y)$ from the system state $x$ : visual cues (lateral position, heading) and proprioceptive cues (power steering).

In the perceptual model, it is assumed that the driver perceives information with a certain inaccuracy and with a given delay. This part contains a lumped time delay and a neuro-motor component. Other components are perception and indifference thresholds, an overall attention level, and attention sharing concepts.

The information processing model contains an internal representation of the system, a 'mental model', based on which the driver estimates the system state.

The control response model determines the driver output, based on the estimated system state and the optimal feedback gains, i.e., feedback gains such that a performance index is minimised.

\section{Results}

\subsection{Initial results}

The overall results of the simulator and the driver model are summarised in Fig. 2. Looking at the simulator results, we found a clear effect of error amplitude: the maximum path and steering deviations are larger for the $200 \mathrm{~ms}$ error than for the $50 \mathrm{~ms}$ error. The driver model results were in the same order of magnitude as the driving simulator results, showing a similar effect of pulse duration.

The effect of error type differed between the model and the simulator. In the driver model, the error type had no effect. In contrast, the driving simulator results showed that changing the error type from 'Torque' to 'No torque' gave an increase of the maximum path deviation, and a decrease of the steering amplitudes. Looking more closely to the short $(50 \mathrm{~ms})$ error, the model results typically exhibited a somewhat smaller path deviation and somewhat more steering activity. .

Based on these results a plausible driver model parameter adjustment will be discussed in the next section. 

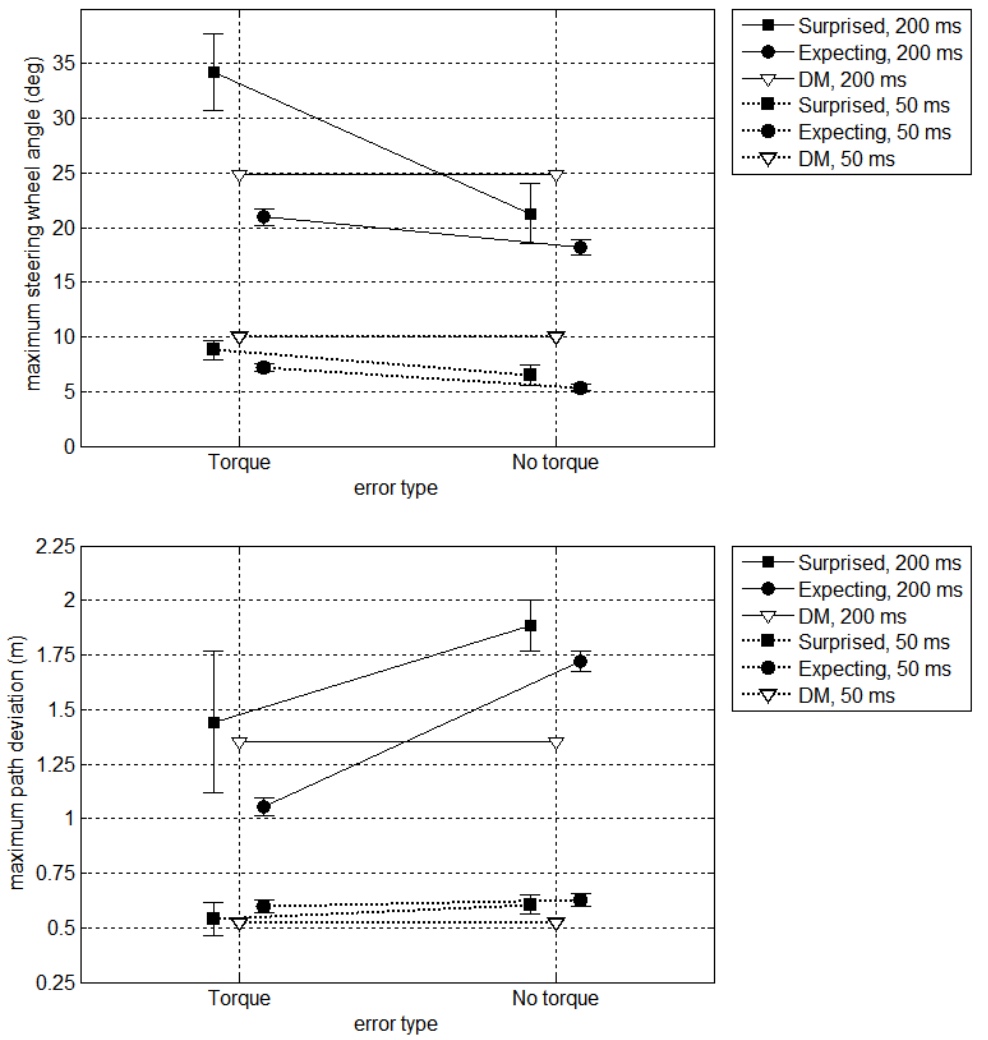

Fig. 2. Maximum steering wheel angle and path deviation (initial results): driving simulator (mean and SE) and driver model (DM) results as a function of error type and pulse duration. .

\subsection{Model matching}

A limited attempt was made to improve the agreement between the model and experimental results. As discussed in the previous section, the model results typically exhibit a somewhat smaller path deviation and somewhat more steering activity for the short $(50 \mathrm{~ms}$ ) errors. This suggests one possible discrepancy between model and experiment: the driver model adopts a slightly different trade-off between the control response and lateral position. This hypothesis has been investigated, but did not result in a clearly different response.

Another explanation could be that the effective moment of inertia of the steering wheel, as reflected by the parameter $\mathrm{K}_{1}$. This parameter can be interpreted as 
the inverse of the moment of inertia of the steering wheel system. In the driving simulator, open-loop tests yielded a value for $\mathrm{K}_{1}$ of $800\left(\mathrm{deg} / \mathrm{s}^{2} / \mathrm{Nm}\right)$. However, in case of the closed loop situation, the driver holds (somehow) the steering wheel, and the effective (overall) moment of inertia will then be larger. Based on preliminary closed-loop tests, a value of $\mathrm{K}_{1}$ of $100 \mathrm{deg} / \mathrm{s}^{2} / \mathrm{Nm}$ was selected. Looking at the current results, this value should possibly be somewhat smaller (corresponding with 'holding the steering wheel somewhat tighter'). A value of $50 \mathrm{deg} / \mathrm{s}^{2} / \mathrm{Nm}$ was considered for the 'No torque' error type. For the error type with Torque, it seemed that the force feedback, combined with the opposite yaw rate, 'encouraged' the driver to respond somewhat quicker (at least for the participants who were expecting the errors, and who were thus, to some extent, 'used' to this condition). In driver model terms: the driver is willing to move the steering wheel somewhat quicker, i.e. a somewhat larger value of the parameter $\mathrm{K}_{1}$. Therefore, the original value of $\mathrm{K}_{1}=100 \mathrm{deg} / \mathrm{s}^{2} / \mathrm{Nm}$ was maintained here.

Furthermore, a somewhat smaller neuromotor time constant $\left(\mathrm{T}_{\mathrm{N}}\right)$ was assumed. Also this adjustment is plausible for the present simulation of suddenly occurring system errors, requiring a rapid driver response. Therefore, a value of $0.12 \mathrm{~s}$ is assumed for all configurations instead of the default value $0.15 \mathrm{~s}$.

Fig. 3 shows that now the agreement between the model and the experimental results are generally better. A closer match to the experimental results could possibly be obtained, e.g., by adjusting the reaction time. However, as we aim at maximum predictive capability for all configurations rather than an ad hoc model match for individual configurations, no further model adjustments were made. 

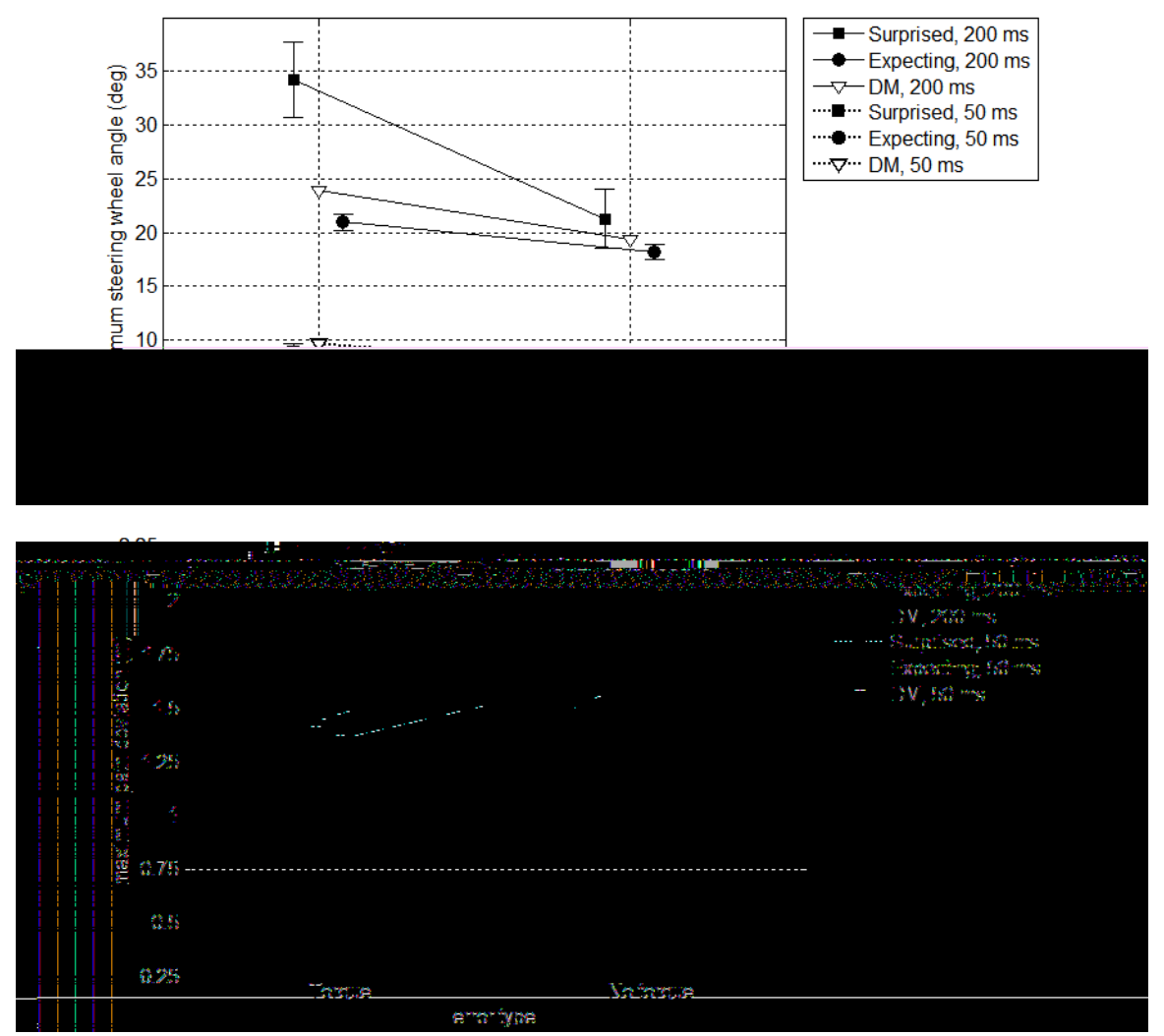

Fig. 3. Maximum steering wheel angle and path deviation (after model matching): driving simulator (mean and SE) and driver model (DM) results as a function of error type and pulse duration.

\section{Discussion and conclusions}

The driver model applied here was originally developed to model the lane keeping task. This model was now applied to investigate how well it could describe the driver's response to various Steer-by-Wire system errors. For various error conditions (two error types, combined with two error pulse durations), the driver model results were predicted and compared with the corresponding experimental results from the driving simulator, using default settings for the driver model parameters.

The (transient) driver response to the considered system errors was analysed in terms of the extreme of the lateral path deviation and of the steering wheel angle. 
Generally, the agreement was relatively good for the $50 \mathrm{~ms}$ pulse configurations, although the model results typically exhibited somewhat smaller path deviation and somewhat more steering activity. For the $200 \mathrm{~ms}$ pulse configurations, the lateral path deviation and steering wheel angle clearly did not match very well (discrepancy was approximately up till $30 \%$ for the lateral path deviation).

In the model matching phase, the values of two plausible model parameters were adjusted based on the comparison between the model predictions and the experimental results. The effective moment of inertia of the steering wheel, as reflected by the parameter $\mathrm{K}_{1}$, was assumed to be somewhat smaller (corresponding with 'holding the steering wheel somewhat tighter') for the error type with no torque. In addition, a somewhat smaller neuromotor time constant was assumed in all conditions. Also this adjustment is plausible for the present simulation of suddenly occurring system errors, requiring a rapid driver response. Using these adjusted driver model parameter values, a rather good match between the model and the experimental results could be obtained.

The general conclusion can be drawn that the overall agreement between the driver model and the experimental results is rather good. Even though the model was originally developed for a stationary lane keeping task, the model has shown to adequately describe driver's response to suddenly occurring system failures and can be used for this type of tasks, providing a useful method to answer a variety of design questions. For example, the predicted relationship between error amplitude, for a given error pulse duration, and acceptable maximum lateral deviation allows the specification of acceptable failure characteristics. Another interesting question is the driver's expectation of failures, which can be investigated in a straightforward manner with the model.

\section{References}

[1] Makowitz, R., \& Temple, C. (2006). FlexRay - A communication network for automotive control systems. IEEE International Workshop on Factory Communication Systems - Proceedings, WFCS. Pp. 207-212. .

[2] Wewerinke, P. H., Hogema, J. H., \& Rook, A. M. (2005). Modelling driver behaviour in the case of failures in a steer-by-wire system (TNO-report TNO-DV3 2005-D007). Soesterberg, The Netherlands: TNO Defence, Security and Safety.

[3] Neukum, A. \& Krüger, H. P. (2003). Fahrerreaktionen bei Lenksystemstörungen - Untersuchungsmethodik und Bewertingskriterien. VDI berichte, 1791 297-318.

[4] Hogema, J. H. (2007). Effects of a failing steer-by-wire system on driving behaviour. Proceed-ings of the 14th world congress on Intelligent Transport Systems, Beijing, 9-13 October 2006.

[5] Kleinman, D. L., Baron, S., \& Levison, W. H. (1970). An optimal control model of human response. Part 1: Theory and validation. Automatica, 6 (3), 357-369.

[6] Wewerinke, P. H. (1989). Models of the human observer and controller of a dynamic system (PhD thesis). Enschede: Universiteit Twente. 